\title{
Hybridly pumped collisional soft X-ray laser in Ne-like sulphur
}

\author{
P.V. Nickles, K.A. Janulewicz, J.J. Rocca' ${ }^{1}$, F. Bortolotto, A. Lucianetti \\ and W. Wandner \\ Max Born Institute, Max-Born-Str. 2 A, 12489 Berlin, Germany \\ ${ }^{1}$ Department of Electrical and Computer Engineering, Colorado State University, Fort Collins, \\ CO 80523, U.S.A.
}

\begin{abstract}
We describe an experiment demonstrating XUV amplification following collisional excitation in a discharge-created plasma waveguide irradiated by a picosecond oplical laser pulse. A capillary discharge was used to generate a plasma radiation pipe with a radially concave electron density profile. Temporally resolved transmission characteristics and guiding effect have been measured. An intense short laser pulse rapidly heated the electrons, producing amplification in the $3 p^{1} S_{0}-3 s{ }^{1} P_{1}$ transition of Ne-like $S$ at $60.84 \mathrm{~nm}$. The integrated gain-length product obtained within a $3 \mathrm{~cm}$ active medium with a laser energy of $0.46 \mathrm{~J}$ was $\sim 6.8$. The pump energy depletion has been analysed as well. This new, hybridly pumped soft $\mathrm{X}$-ray laser with the transient gain offers a new way towards efficient tabletop coherent XUV sources.
\end{abstract}

\section{INTRODUCTION}

A collisionally pumped X-ray laser (XRL) with a transient inversion [1] showed enormous advantages of the double-pulse (long/short) pumping scheme. Its involvement in experimental practice resulted in reduction in the pump energy up to two orders of magnitude and similar scale of reduction in the output pulse length if compared to the quasi-steady-state regime (QSS). Using this pumping method the germanium $(\lambda=19.6 \mathrm{~nm})$ and titanium $(\lambda=32.6 \mathrm{~nm})$ XRLs have been saturated and the gain coefficients of 30 and $35 \mathrm{~cm}^{-1}$, respectively, have been achicved with a moderate pump energy of $10-20 \mathrm{~J}[2,3]$. The total laser pump energy as high as $7 \mathrm{~J}$ caused saturation in $\mathrm{Ni}-$ like $\mathrm{Pd}$ laser $(\lambda=14.7 \mathrm{~nm})$ and gave maximal ever registered gain coefficient of $63 \mathrm{~cm}^{-1}[4]$. However, this robust scheme cannot take the full adventage of its unique properties duc to the refraction effect. Additionally, the short duration time of the gain, caused by heating on the picosecond time scale, supports a short pulse emission in the XUV spectral range but at the same time it requires complicated optical arrangement to ensure efficient cxtraction of the energy deposited in the active medium by the travelling wave pump regime.

On the other hand there exists an alternative, very simple and relatively cheap XRL-pumping scheme based on a very fast electrical discharge through a capillary. A capacitor bank stores an energy of several tens of joules which is rapidly discharged through a capillary filled with gas. The peak current achieves a few tens of kiloamps within $30-50 \mathrm{~ns}$ [5]. Relatively low gain coefficient about $1 \mathrm{~cm}^{-1}$ is compensated by a very long (up to $25-30 \mathrm{~cm}$ ) active medium and saturation of the output has been demonstrated [6]. The major disadvantage of the system working in a quasi-steady-state regime (QSS) is scaling this scheme to shorter wavelengths (at the moment $46.9 \mathrm{~nm}$ in Ne-like Ar), which is a serious technological challenge. The long output pulse $(\sim \mathrm{ns})$ is not favourable in some applications requiring high temporal resolution. However, this system is able to work with an increased (up to $7 \mathrm{~Hz}$ ) repetition rate and delivers average energy of $0.88 \mathrm{~mJ}(\mathrm{P} \sim 3 \mathrm{~mW})$ with the repetition rate of $4 \mathrm{~Hz}$ [7].

It is easily seen from the above that both schemes discussed are complementary to some extent. Meaning the advantages of one fit very well the disadvantages of the second. Capillary discharge is a very simple, compact and cheap source of plasma with an abundance of the Ne-like ions. Hence, this is 
potentially an excellent source of preformed plasma for the transient inversion pumping method and it could replace the whole optical laser system generating the nanosecond pulse. The short, guided, picosecond pulse would heat rapidly the plasma created by the discharge. Moreover, quasi-travelling wave pumping is inherent for this scheme. Additionally, the high intensity short laser pulse could easily improve the plasma ionization stage by the field ionization. Guiding in a symmetric plasma pipe reduces the problems present in the conventional scheme due to refraction. Recently, a proposal to combine both schemes in one hybrid laser system appeared and was accompanied by the initial numerical simulations [8].

\section{EXPERIMENTAL SETUP}

Here we present the results of an experiment on a capillary if irradiated by the short CPA laser pulse. Sulphur capillaries with lengths between 10 and $30 \mathrm{~mm}$ and channel diameter of $0.5 \mathrm{~mm}$ and $1 \mathrm{~mm}$ have been used in the measurements. The capillary with broader channel gave no gain and will not be discussed further. The laser pulse, used in the experiment described, had length of $2 \mathrm{ps}$ and delivered energy between 0.1 and $1.0 \mathrm{~J}$ from the MBI glass laser system $(\max .10 \mathrm{~J}, 1.05 \mu \mathrm{m}$ ). The laser beam was focused to a diameter of $170 \mu \mathrm{m}$ (FWHM). The plasma was created by ablation of the wall material during the discharge. The peak discharge current, measured with a Rogowski coil, was kept about $3 \mathrm{kA}$, while the FWHM of the current first half-cycle was $\sim 150$ ns. Sulphur was used in this proof-ofprinciple experiment as a lasant because its level structure is especially suitable for transient pumping, and a gain coefficient of $0.45 \mathrm{~cm}^{-1}$ was recently reported on the $3 \mathrm{p}-3 \mathrm{~s}$ transition in Ne-like sulfur at 60.8 $\mathrm{nm}$ when a pure capillary discharge was used as a pump [9]. The transmitted energy and beam shape of the short pumping pulse were measured using the experimental set-up shown in Fig. 1. An 8-bit CCD camera and a pyroelectric calorimeter were used to monitor the beam shape a few millimeter after the capillary exit and measure the output energy, respectively. Glass wedges and a set of the neutral density filters (NG) were used to attenuate the incident beam. The beam shape was modified slightly by three diaphragms installed behind the capillary exit to reduce the debris and make possible a differential vacuum pumping scheme.

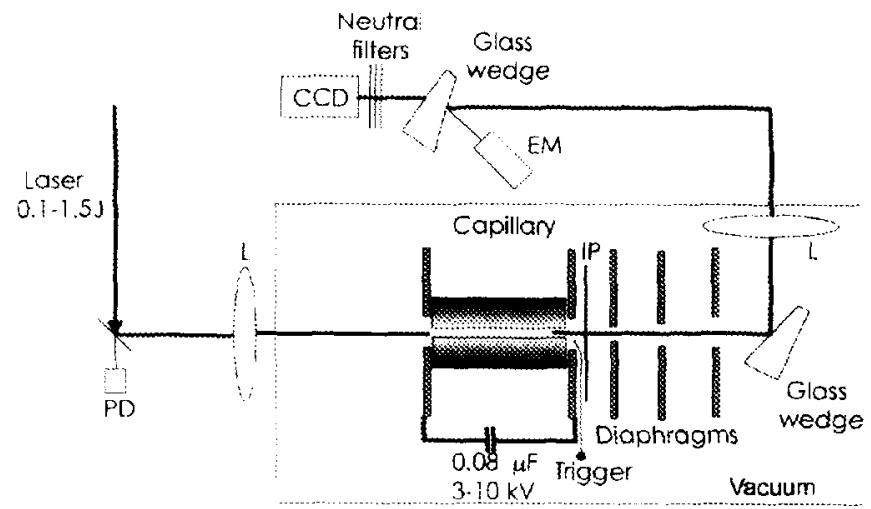

Figure 1. Experimental setup for transmission measurement and beam monitoring.

\section{RESULTS}

The main aim of the laser transmission experiment was to determine the time gap in which the short pumping pulse heats the plasma due to the IB absorption but still shows a finite transmission to avoid a cold plasma at the capillary end. The guiding effect caused by the concave radial density profile of the plasma with a minimum on the axis should facilitate efficient heating along the active medium.

\subsection{Laser beam transmission}

The transmission curve for $10 \mathrm{~mm}$ capil discharge current waveform in Fig.2. Th the moment of the laser pulse arrival. Th always calculated in respect to this mor lengths used.
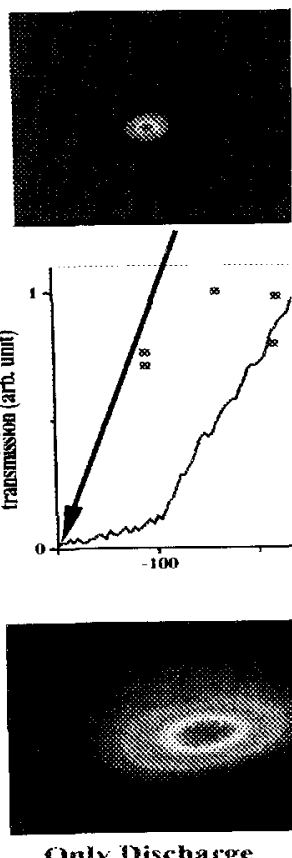

Figure 2. Transmission of the capillary disc intensity distributions in the transmitt

This is a consequence of the constant ablation process and the plasma dynan the time of the current maximum and which no laser is transmitted through emission) has been estimated to last discharge parameters. The beam sha from the beam image when the capilla full opacity phase after the current pe transparency phase. The plasma dens the capillary axis, allowing for the prop the transmission phase. It is worth $r$ significantly affect the regular and transmission character. The guiding $p$ at $35 \mathrm{~ns}$. The plasma emission maxin 


\subsection{Laser beam transmission}

and it uided, elling easily duces both ations

pulse. have lot be vered $n$ was iterial $3 \mathrm{kA}$, f-ofiping, $: 60.8$ pe of $\mathrm{CCD}$ ir the insity three sntial

The transmission curve for $10 \mathrm{~mm}$ capillary is presented together with the beam shape images and the discharge current waveform in Fig.2. The beam images are related to the instantaneous current value at the moment of the laser pulse arrival. The peak current position is a reference and the laser pulse delay is always calculated in respect to this moment. The transmission behaviour was similar for all capillary lengths used.

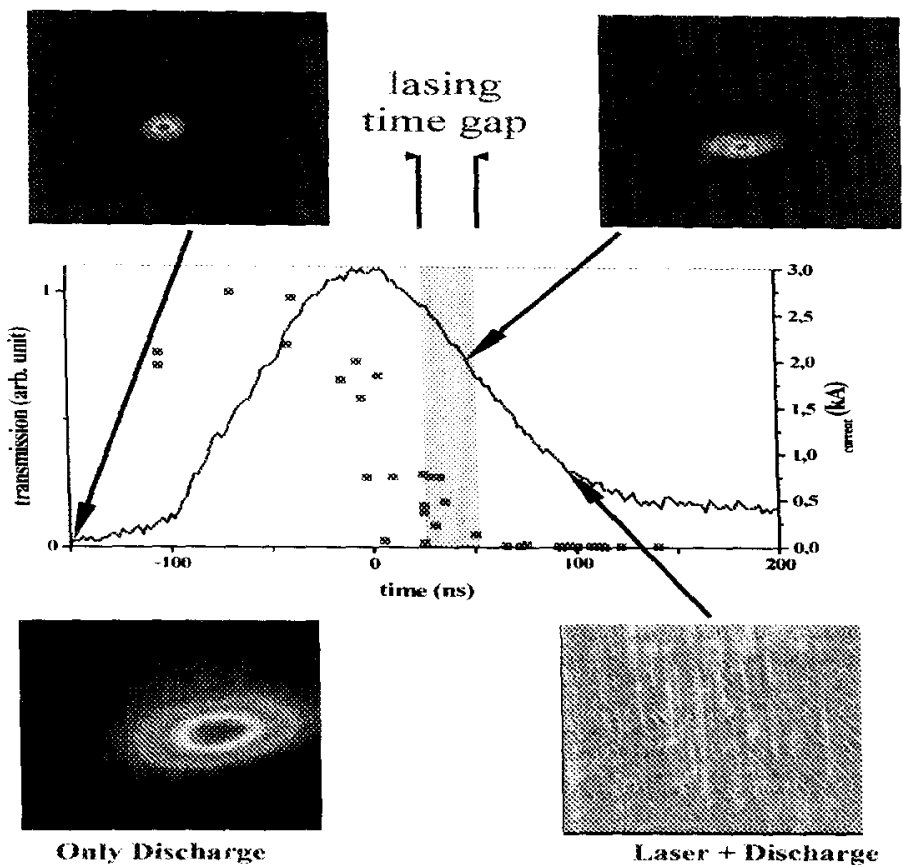

Discharge emission blocked by hilter

Figure 2. Transmission of the capillary discharge plasma irradiated by a picosecond laser pulse, current waveform and intensity distributions in the transmitted laser beam. Capillary length $10 \mathrm{~mm}$. Intesity distributions:

This is a consequence of the constant current value used in the experiment, as the current determines the ablation process and the plasma dynamics. There is an abrupt fall in the transmission which begins near the time of the current maximum and lasts $80-90 \mathrm{~ns}$. The duration of the following opacity period (in which no laser is transmitted through the plasma column and the image is determined by the plasma emission) has been estimated to last between 6 and $13 \mu$ sepending on the capillary dimensions and discharge parameters. The beam shapes registered cover the whole history of the transmission phase from the beam image when the capillary is irradiated by the laser before the discharge triggering to the full opacity phase after the current peak. The beam shape is highly symmetric during nearly the whole transparency phase. The plasma density distribution becomes symmetric and with minimum density on the capillary axis, allowing for the propagation of the symmetric laser beam profile observed at the end of the transmission phase. It is worth noting that the plasma ablated from the capillary wall does not significantly affect the regular and symmetric shape of the laser beam but significantly changes the transmission character. The guiding phase was observed for delays between 25 and 60 ns with optimum at $35 \mathrm{~ns}$. The plasma emission maximum in VIS spectral range, registered with a fast photodiode, was

iid a 
delayed about $68 \mathrm{~ns}$ relative to the current waveform peak. The temporal history of this emission was measured using Hadland IMACON 500 streak camera and the result is shown in Fig.3a. We have traced two cross sections of the registered streak which were positioned 29 and 44 ns after the current peak (Fig.3b). Having assumed proportionality between the emitted light intensity and density of the the emitting ions we have found that a distinctly concave and symmetric density profile exists 29 ns after the current peak and this becomes much more weakly pronounced 15 ns later. The depth of the plasma channel corresponds to the density reduction about $27 \%$. These values correspond reasonably to our observation that the best lasing results were obtained about $35 \mathrm{~ns}$ after the current maximum and the channel depth expected is between 40 and $60 \%$.

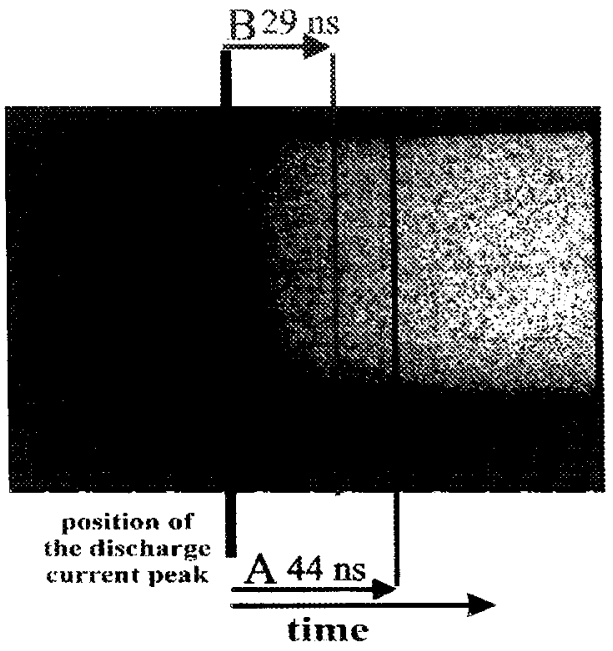

a)
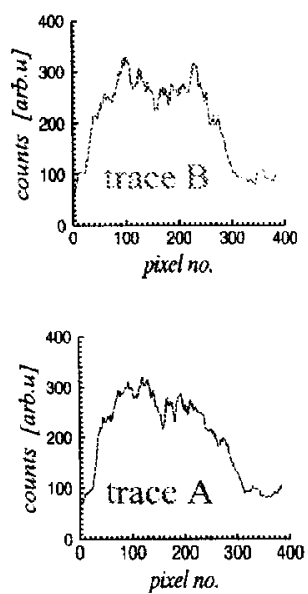

VIS-emission protiles

b)
Figure 3. Temporal dependence of the plasma emission in the visible range of the spectrum relative to the current waveform; a) streak camera image with the marked current peak position; b) streak cross sections showing radial distribution of the plasma VIS-emission, which is proportional to the ion density.

\subsection{Spectral measurements}

The spectral diagnostic consisted of a flat-field spectrograph (FFS) provided with a 1200 lines/mm Alcoated, Harada-type concave diffraction grating. The radiation dispersed by the grating was recorded by a sensor consisting of a double-stage MCP, phosphor screen and a 16-bit CCD detector. The MCP was gated with a temporal window of $40 \mathrm{~ns}$ at the flat-top. The input slit of the spectrograph had a width of $100 \mu \mathrm{m}$. This setup allowed for relatively poor spectral resolution at the wavelength of interest, as the optimal wavelength range for such a grating is far from $600 \AA(5.0-30.0 \mathrm{~nm})$. No filters were installed due to the limited amount of radiation available. It is seen from the spectrum in Fig. 4 that the pump laser radiation, at the energy level used, strongly affects the spectrum at $60.8 \mathrm{~nm}$. The $60.84 \mathrm{~nm}$ line of Nelike sulfur (S VII) is intensified significantly by the irradiation while the other lines are hardly changed. This influence changes nonlinearly with the capillary length (Fig.5)and there is no doubt that the intensity increase of the $60.84 \mathrm{~nm}$ line is caused by the laser plasma heating. We estimated the gain coefficient from the data presented in Fig.6. These data is influenced by non-uniform heating in the axial direction. Given a realistic value of $2 \times 10^{19} \mathrm{~cm}^{-3}$ for the electron density reported earlier in other experiments on the capillary discharge plasma [10-12] and an initial electron temperature of $10 \mathrm{eV}$ we estimated the contribution of the inverse bremsstrahlung absorption using analytic formulae derived by Pert [13]. These estimates correspond to the plasma temperature on the capillary axis and do not take the pump laser beam profile into account. The results well as a subsequent non-unifor

Figure 4. The spectra of the sulphur 1 line) and discharge irradiated

Under these circumstaces the no more valid. We divided th determined the gain coefficient been obtained for the $10 \mathrm{~mm}$ ca $1.2 \mathrm{~cm}^{-1}$, respectively. The effec confirmed we observed lasing. estimate roughly the pump sche the collisionally pumped XRLs. pump energy taken to this estim no direct proof yet, that we obse significantly stronger than that scheme with a long laser pulse character of the gain.

Figure 5. Maximum output signal vs 
sion was ve traced ent peak f the the after the plasma $y$ to our and the m Alded by 工P was idth of as the istalled p laser of $\mathrm{Ne}-$ langed. Itensity fficient ection. , on the ted the . These sr bcam profile into account. The results showed strong pump energy depletion along the capillary axis (30\%) as well as a subsequent non-uniform electron energy distribution with $10 \%$ reduction in the axial direction.

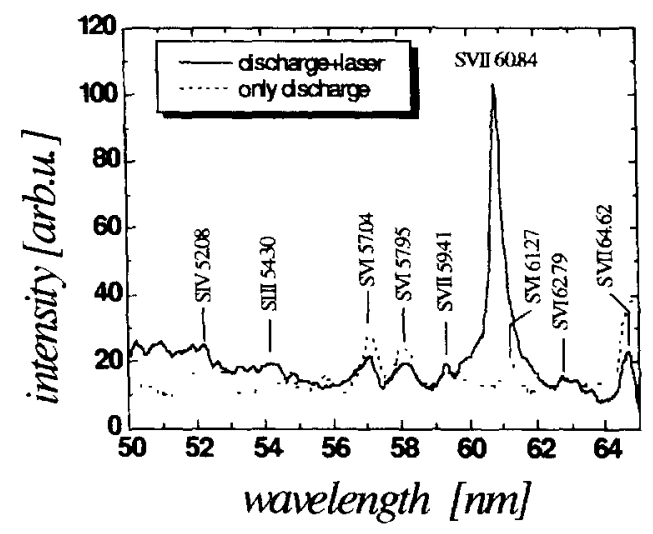

Figure 4. The spectra of the sulphur plasma measured at $\sim 35 \mathrm{~ns}$ delay relative to the current peak for pure discharge (dotted line) and discharge irradiated by a picosecond laser pulse. The capillary length was $30 \mathrm{~mm}$.

Under these circumstaces the traditional methods of gain estimation, for example Linford formula, are no more valid. We divided the capillary into three separate sections, each with a $1 \mathrm{~cm}$ length and determined the gain coefficient for each section separately. The maximum gain, equal to $4.6 \mathrm{~cm}^{-1}$, has been obtained for the $10 \mathrm{~mm}$ capillary. The second and third section had the gain coefficients of 1.0 and $1.2 \mathrm{~cm}^{-1}$, respectively. The effective gain-length product estimated for all sections was equal to 6.8 and confirmed we observed lasing. The ratio (gain-length product $\mathrm{gl}$ )/(pump energy $\mathrm{E}_{\mathrm{p}}$ ) commonly used to estimate roughly the pump scheme efficiency is equal to $3.1 \mathrm{~J}^{-1}$ and belongs to the highest obtained for the collisionally pumped XRLs. Typical values spread between 1 and $2 \mathrm{~J}^{-1}$. It is worth noting that the pump energy taken to this estimate includes the total electric energy dissipated in the discharge. There is no direct proof yet, that we observed transient gain. However, one can argue that the signal measured is significantly stronger than that obtained in the pure electric discharge plasma and within the prepulse scheme with a long laser pulse with energy of $20 \mathrm{~J}$ [14]. The reason for this could be the transient character of the gain.

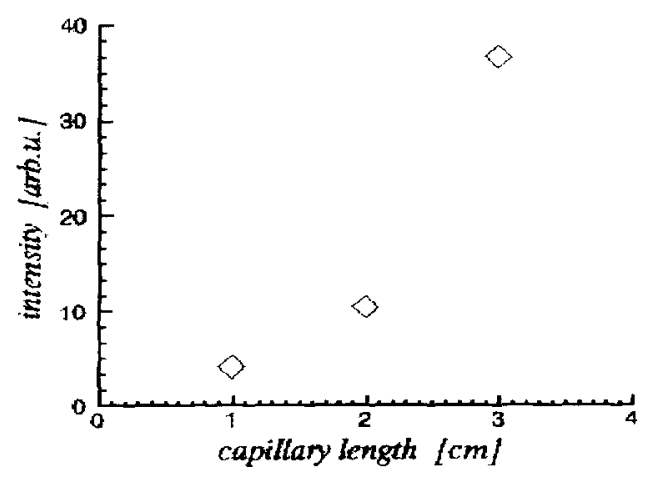

Figure 5. Maximum output signal vs. the capillary length. 


\section{CONCLUSIONS}

We have irradiated a capillary discharge with a picosecond laser pulse of relatively low energy (320-460 $\mathrm{mJ}$ ) and estimated the temporal dependence of the transmission as well as monitored the shape of the beam transmitted through the capillary channel during the discharge. The transmission decreases very quickly immediately after the current peak during the next $80-90$ ns and the beam shape is evidently symmetrised with clear indication of the guiding effect. The corresponding spectra show significant signal increase with the medium length at $60.84 \mathrm{~nm}$. We ascribe this increase to fast plasma heating by the picosecond pulse followed by lasing on the $3 \mathrm{p}-3 \mathrm{~s}$ transition in Ne-like sulphur. The results presented are encouraging and promise a new efficient tabletop hybrid $X$-ray laser scheme. Other materials containing elements with higher atomic number $Z$, which are required to shorten the emitted wavelength, are under investigation.

\section{Acknowledgments}

This work was supported by DFG (Deutsche Forschungs Gemeinschaft) and European X-Ray Laser Network and U.S. National Science Foundation.

\section{References}

[1] Nickles P.V., Shlyaptsev V.N., Kalachnikov M.,Schnürer M.,Will I. and Sandner W., Phys. Rev. Lett. 78 (1997) 2748

[2] P.J.Warwick et al., J. Opt. Soc. Am. B 15 (1998) 1808-1814

[3] M.P. Kalachnikov et al., Phys. Rev. A 57 (1998) 4778

[4] J. Dunn et al., "Tabletop transient collisional excitation $x$-ray lasers", Soft X-Ray Lasers and Applications III, Denver 19-20 July 1999, J.J. Rocca and L.B. Da Silva Eds. (Proceedings of SPIE 3776) pp. 2-8

[5] Rocca J.J., Shlyaptsev V.N., Tomasel F.G., Cortazar O.D., Hartshorn D., Chilla J.L.A., Phys. Rev. Lett. 73 (1994) 2192

[6] Rocca J.J., Clark D.P., Chilla J.L.A. and Shlyaptsev V.N., Phys. Rev. Lett. 77 (1996) 1476

[7] Benware B.R., Machictto C.D., Moreno C.H. and Rocca J.J., Phys. Rev. Lett. 81 (1998) 5804

[8] Osterheld A.L. ct al., "Modelling of Laser Produced Plasma and Z-Pinch X-Ray Lasers", 6th International Conference on X-Ray Lasers and Applications, Kyoto $31^{\text {th }}$ Aug $-4^{\text {th }}$ Sept 1998, Y. Kato, H. Takuma and H. Daido Eds. (IOP Conf. Ser. 159, IOP Publishing) pp. 353-362

[9] Tomasel F.G., Rocca J.J., Shlyaptsev V.N. and Macchictto C.D., Phys. Rev. A 55 (1997) 1437

[10] Rocca J.J. et al., "New Results in the Development of Table-Top Capillary Discharge Soft XRay Lasers: Demonstration of High Average Power and Realization of the First Demonstration", 6th International Conference on X-Ray Lasers and Applications, Kyoto 31 ${ }^{\text {th }}$ Aug $-4^{\text {th }}$ Sept 1998, Y. Kato, H. Takuma and H. Daido Eds. (IOP Conf. Ser. 159, IOP Publishing Bristol) pp. 9-16

[11] Rocca J.J., Marconi M.C., and Tomasel F.G., IEEE J. of Quant. Electronics 29 (1993) 182

[12] Tomasel F.G., Rocca J.J., Cortazar O.D., Szapiro B.T., and Lee R.W., Phys. Rev. E 47 (1993) 3590

[13] Pert G.J., Phys. Rev. E 51 (1995) 4778

[14] Li Y., Pretzler G., Lu P., Nilsen J. and Fill E., "Ne- and Ni-like $\mathrm{x}$-ray lasers using the prepulse technique", 5th International Conference on X-Ray Lasers and Applications, Lund 10-14 June 1996, S. Svanberg and C.-G. Wahlström Eds. (IOP Conf. Ser. 151, IOP Publishing Bristol) pp. 255-259

\section{Investigating the dynami leads to soft X-ray laser}

\author{
A. Ben-Kish, M. Shuker, R.A. \\ and J.L. Schwob \\ Physics Department, Technion - Is \\ ${ }^{1}$ Racach Institute of Physics, The I
}

\section{INTRODUCTION}

In recent studies conducted by Rocca Ar and $S$ was obtained by means of a $\mathrm{Z}$-pinch device, stable and uniform $\mathrm{c}$ Furthermore, specific plasma conditio This includes, for example in the Netemperature ( $\mathrm{Te}=50-80 \mathrm{eV}$ ) and dens achieving beam guiding and low opaci

In this paper we present: First, the the collapsing column in a capillary column; Third, the plasma conditions from the soft $x$-ray spectrum emitted b scheme allowed us to control the plas where strong amplification at $46.9 \mathrm{~nm}$

\section{INVESTIGATING THE COLUI}

Our fast capillary discharge setup [3 consisting of a capillary tube $(80 \mathrm{~m}$ Subsequent to a pre-ionizing pulse in generating the main current pulse ( $\mathrm{I}_{\mathrm{mai}}$

The appropriate initial condition dynamics [4] were previously investig this scheme, a new detection system developed. In this detection system, imaging of the compressed column is $50 \mathrm{~mm}$ from the capillary. Shallow imaging the entire, $80 \mathrm{~mm}$ long, plasi The recording medium, located $370 \mathrm{~m}$ ray Image Plate [6] or a gated (2-5ns 